\title{
Kinetics of many-body reservoir engineering
}

\author{
Hugo Ribeiro ${ }^{1}$ and Florian Marquardt ${ }^{1,2}$ \\ ${ }^{1}$ Max Planck Institute for the Science of Light, Staudtstraße 2, 91058 Erlangen, Germany \\ ${ }^{2}$ Institute for Theoretical Physics, Department of Physics, University of Erlangen-Nürnberg, Staudtstrasse 7, 91058 Erlangen, Germany
}

(Received 17 December 2019; accepted 24 July 2020; published 10 August 2020)

\begin{abstract}
Recent advances illustrate the power of reservoir engineering in applications to many-body systems, such as quantum simulators based on superconducting circuits. We present a framework based on kinetic equations and noise spectra that can be used to understand both the transient and long-time dynamics of many particles coupled to an engineered reservoir in a number-conserving way. For the example of a bosonic array, we show that the nonequilibrium steady state can be expressed, in a wide parameter regime, in terms of a modified Bose-Einstein distribution with an energy-dependent temperature.
\end{abstract}

DOI: 10.1103/PhysRevResearch.2.033231

\section{INTRODUCTION}

Reservoir engineering [1-3] is used to deliberately generate some desired dissipative dynamics, as demonstrated in a variety of platforms: atoms [4], superconducting circuits [5-7], ion traps [8-10], and optomechanics [11,12]. In the future, it could become particularly useful for controlling quantum many-body systems, as theoretically proposed in several works (see, e.g., Refs. [13-18]). In this context, an important scenario concerns the case where the coupling to the reservoir conserves the total number of particles [19]. In that way, reservoir engineering contributes to the implementation of quantum simulators, especially in cases where the naturally available dissipation would not drive the system to the right many-body ground state.

The first experimental realizations of such particleconserving reservoir engineered many-body systems are starting to appear: It has been used to stabilize a circuit QEDbased Mott insulator in a one-dimensional (1D) chain of eight transmon qubits against photon losses [20] as well as to dissipatively prepare quantum states in a three-transmon array [19]. However, a simple but yet quantitative theory is missing to describe both the transient and long-time dynamics of such experiments.

Here, we introduce a framework to quantitatively describe the kinetics of many particles being scattered among states due to the interaction with an arbitrary nonequilibrium reservoir [see Fig. 1(a)]. Our theory allows one to understand both the transient and long-time behavior. In particular, we establish a perturbative approach to derive the steady-state distribution in momentum space. Since we are dealing with

Published by the American Physical Society under the terms of the Creative Commons Attribution 4.0 International license. Further distribution of this work must maintain attribution to the author(s) and the published article's title, journal citation, and DOI. Open access publication funded by the Max Planck Society. a nonequilibrium system, the steady-state distribution will depend on details of the interaction and of the reservoir noise spectrum, in contrast to the case of a thermal heat bath encountered, e.g., in certain approaches on particle-conserving photon equilibration [21-23].

The physics we encounter is partially reminiscent of cavity optomechanics [24], except that we are now dealing with a many-particle system instead of a mechanical resonator. Furthermore, the coupling to that system has been engineered to preserve the number of excitations, somewhat analogous to the unconventional quadratic coupling encountered in some optomechanical systems [25].

As an example, we derive the steady state of a 1D array of bosonic modes with nearest-neighbor hopping coupled to a particle-conserving nonequilibrium reservoir. This turns out to be a "deformed" Bose-Einstein distribution with an energydependent effective temperature. We furthermore observe features such as negative-temperature states and prominent accumulation of particles at certain momenta during the time evolution.

We stress that while we focus on the experimentally relevant case of circuit QED, our framework is platform independent. As such, it can be used to predict the time dynamics of other implementations of a many-body system coupled to an engineered reservoir in a number-conserving way, e.g., optomechanical arrays consisting of a chain of mechanical modes, each of which is coupled quadratically [25] to a driven cavity or cold atoms in an optical lattice coupled to a Bogoliubov reservoir [13].

\section{MODEL}

We consider a general situation of bosons hopping on a $d$-dimensional lattice. Each lattice site is coupled to an engineered reservoir via a local density-density interaction [see Fig. 1(b)]. We further assume that the reservoir is kept in a nonequilibrium state, e.g., a steady state resulting from a competition between an external drive and dissipation. The 

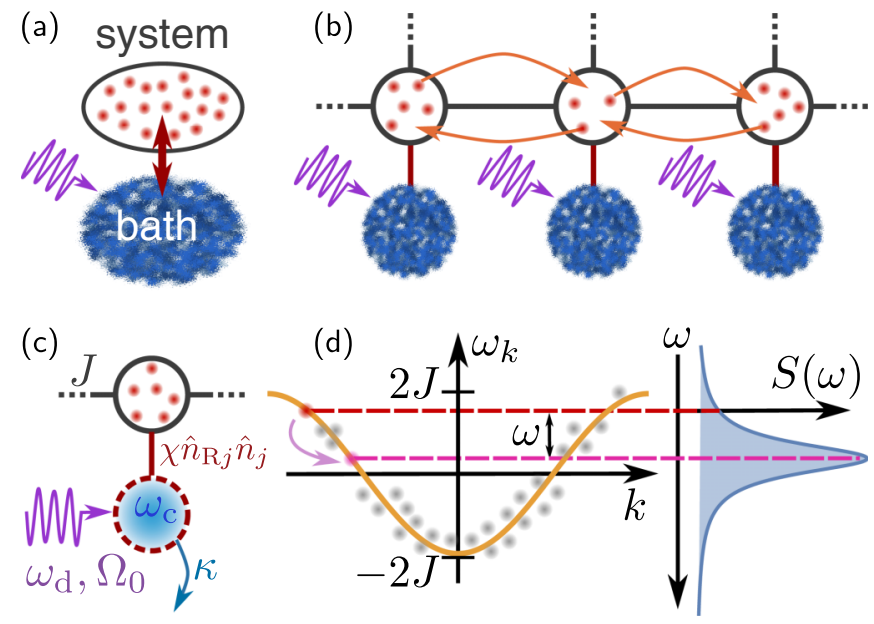

FIG. 1. Many-body reservoir engineering. (a) Many-particle system coupled in a particle-conserving manner to a nonequilibrium bath. (b) Array of bosonic modes, where each site experiences a density-density coupling to a nonequilibrium reservoir. (c) In a cQED setup, a driven, lossy cavity implements a nonequilibrium reservoir. (d) A particle at energy $\omega_{1}$ is scattered to a state of energy $\omega_{2}<\omega_{1}$ by emitting a photon into the reservoir. The rate at which particles scatter is proportional to the noise spectrum $S(\omega)$ of the reservoir.

total Hamiltonian describing this situation can be written as

$$
\hat{H}(t)=\hat{H}_{\text {array }}+\hat{H}_{\mathrm{R}}(t)+\hat{H}_{\text {int }},
$$

where $\hat{H}_{\text {array }}$ is the quadratic Hamiltonian describing the bosonic modes and we denote the annihilation operator of mode $\boldsymbol{r}$ by $\hat{b}_{\boldsymbol{r}}$. The Hamiltonian $\hat{H}_{\mathrm{R}}(t)$ describes the nonequilibrium reservoir and we denote the annihilation of a reservoir excitation by $\hat{R}_{\boldsymbol{r}}$. Most importantly, we assume a densitydensity coupling between the array and the reservoir, of the form

$$
\hat{H}_{\mathrm{int}}=\chi \sum_{r} \hat{R}_{r}^{\dagger} \hat{R}_{\boldsymbol{r}} \hat{b}_{r}^{\dagger} \hat{b}_{\boldsymbol{r}}
$$

with $\chi$ being the coupling constant. Alternatively, one can consider a situation where only one site of the lattice is coupled to a nonequilibrium reservoir. This setting leads qualitatively to the same dynamics. However, the situation studied here presents the advantage of being homogeneous and having a well-defined thermodynamic limit, and the cooling power scales with the size of the system.

To understand how $\hat{H}_{\text {int }}$ gives rise to particle-conserving dissipation processes, it is useful to work in the frame that diagonalizes $\hat{H}_{\text {array }}$. We introduce the mode operators $\hat{\beta}_{k}$ with energy $\omega_{k}$ and defined via the relation $\hat{b}_{\boldsymbol{r}}=\sum_{\boldsymbol{k}} \varphi_{\boldsymbol{r}}(\boldsymbol{k}) \hat{\beta}_{\boldsymbol{k}}$, where $\varphi_{\boldsymbol{r}}(\boldsymbol{k})$ is the mode function, indexed by a label $\boldsymbol{k}$ (in a translational invariant system $\boldsymbol{k}$ would be the wave vector). The interaction Hamiltonian is then given by

$$
\hat{H}_{\mathrm{int}, \mathrm{D}}=\chi \sum_{\boldsymbol{r}, \boldsymbol{k}, \boldsymbol{k}^{\prime}} \varphi_{\boldsymbol{r}}^{*}(\boldsymbol{k}) \varphi_{\boldsymbol{r}}\left(\boldsymbol{k}^{\prime}\right) \hat{R}_{\boldsymbol{r}}^{\dagger} \hat{R}_{\boldsymbol{r}} \hat{\beta}_{\boldsymbol{k}}^{\dagger} \hat{\beta}_{\boldsymbol{k}^{\prime}} .
$$

Using Fermi's golden rule, one can derive transition rates from mode $\boldsymbol{k}$ to $\boldsymbol{k}^{\prime}$,

$$
\Gamma_{\boldsymbol{k} \rightarrow \boldsymbol{k}^{\prime}}=\chi^{2} \gamma_{\boldsymbol{k}, \boldsymbol{k}^{\prime}} S\left(\omega_{\boldsymbol{k} \boldsymbol{k}^{\prime}}\right) n_{\boldsymbol{k}}\left(n_{\boldsymbol{k}^{\prime}}+1\right)
$$

where $\omega_{\boldsymbol{k} \boldsymbol{k}^{\prime}}=\omega_{\boldsymbol{k}}-\omega_{\boldsymbol{k}^{\prime}}$, and we introduce the power spectrum of the nonequilibrium reservoir noise,

$$
S(\omega)=\int_{-\infty}^{\infty} d t \exp (i \omega t)\langle(\delta \hat{n}(t) \delta \hat{n}(0)\rangle,
$$

with $\delta \hat{n}(t)=\hat{R}^{\dagger}(t) \hat{R}(t)-\left\langle\hat{R}^{\dagger}(t) \hat{R}(t)\right\rangle$, and we define

$$
\gamma_{\boldsymbol{k}, \boldsymbol{k}^{\prime}}=\sum_{\boldsymbol{r}}\left|\varphi_{\boldsymbol{r}}^{*}(\boldsymbol{k})\right|^{2}\left|\varphi_{\boldsymbol{r}}\left(\boldsymbol{k}^{\prime}\right)\right|^{2} .
$$

Here, we assumed for simplicity independent reservoirs with identical properties, although this assumption could easily be dropped.

Now we are in a position to describe the dynamics of the array, by formulating a set of coupled kinetic equations that describe the evolution of the average occupation of the eigenmodes of the array:

$$
\dot{n}\left(\omega_{\boldsymbol{k}}, t\right)=\sum_{\boldsymbol{k}^{\prime} \neq \boldsymbol{k}}\left[\Gamma_{\boldsymbol{k}^{\prime} \rightarrow \boldsymbol{k}}(t)-\Gamma_{\boldsymbol{k} \rightarrow \boldsymbol{k}^{\prime}}(t)\right],
$$

with $n\left(\omega_{k}, t\right)=\left\langle\hat{\beta}_{k}^{\dagger}(t) \hat{\beta}_{k}(t)\right\rangle$. In the presence of weak interactions between particles, one would have to supplement Eq. (7) by addition of two-particle scattering terms [26].

An important aim in any nonequilibrium scenario is to characterize the resulting steady-state distributions. The continuum limit of Eq. (7),

$$
\begin{aligned}
\dot{n}\left(\omega_{\boldsymbol{k}}, t\right)= & \int_{\omega_{\min }}^{\omega_{\max }} d \omega_{\boldsymbol{k}^{\prime}} D\left(\omega_{\boldsymbol{k}^{\prime}}\right)\left\{S\left(\omega_{\boldsymbol{k}^{\prime} \boldsymbol{k}}\right) n\left(\omega_{\boldsymbol{k}^{\prime}}\right)\left[n\left(\omega_{\boldsymbol{k}}\right)+1\right]\right. \\
& \left.-S\left(-\omega_{\boldsymbol{k}^{\prime} \boldsymbol{k}}\right) n\left(\omega_{\boldsymbol{k}}\right)\left[n\left(\omega_{\boldsymbol{k}^{\prime}}\right)+1\right]\right\},
\end{aligned}
$$

where $D\left(\omega_{k}\right)$ denotes the density of states, is particularly useful to find perturbative solutions describing the steady state of the system. To understand how this can be done, we start by recalling that even for a noise source that is not in thermal equilibrium one can always define an effective temperature associated to a single transition frequency by using the Stokes relation $S(\omega) / S(-\omega)=\exp \left[\beta_{\mathrm{eff}} \omega\right]$ or, alternatively, $\tanh \left[\beta_{\text {eff }} \omega / 2\right]=[S(\omega)-S(-\omega)] /[S(\omega)+S(-\omega)]$ [27]. By expanding on both sides of the equality and noting that the expression on the right-hand side is an odd function of $\omega$ $(S(\omega)$ is an asymmetric function of $\omega$ [27]), we find that the effective inverse temperature for a single transition frequency can be expressed as $\beta_{\text {eff }}(\omega)=\sum_{n=0} \beta_{2 n} \omega^{2 n}$. In the limit where $\beta_{2 n} \omega^{2 n} / \beta_{0} \ll 1$ with $n \geqslant 1$, the reservoir effectively acts as a thermal reservoir since all transitions have the same the temperature $\beta_{0}$, which we define as the equilibrium temperature. Thus, we expect the steady state to be described by Bose-Einstein statistics with an inverse temperature $\beta_{0}$ and a chemical potential $\mu$ :

$$
n_{\mathrm{BE}}\left(\omega_{\boldsymbol{k}}\right)=\frac{1}{\exp \left[\beta_{0}\left(\omega_{\boldsymbol{k}}-\mu\right)\right]-1} .
$$

In the vicinity of this limiting case, we can look for a description in terms of small corrections around the equilibrium statistics, which can be done using a series representation for the steady state:

$$
n^{(\infty)}\left(\omega_{k}\right)=\sum_{i} n_{i}\left(\omega_{k}\right)
$$


with $n_{0}\left(\omega_{\boldsymbol{k}}\right)=n_{\mathrm{BE}}\left(\omega_{\boldsymbol{k}}\right)$ and $n_{i} \propto\left(\beta_{0} \omega_{\boldsymbol{k}}\right)^{i}(i \geqslant 1)$. In other words the steady-state distribution can be understood as a certain deformed version of the Bose-Einstein distribution.

\section{IMPLEMENTATION WITH CQED}

The physical situation described by Eq. (1) can be realized, e.g., using linear elements of the superconducting circuits toolbox. The array can be engineered by capacitively coupling cavities and the local nonequilibrium reservoir can be realized using driven, lossy cavities [see Fig. 1(c)]. In this context, we have

$\hat{H}_{\mathrm{R}}(t)=\omega_{\mathrm{c}} \sum_{r} \hat{a}_{r}^{\dagger} \hat{a}_{r}+\Omega_{0} \sum_{r}\left[\exp \left(-i \omega_{\mathrm{d}} t\right) \hat{a}_{r}^{\dagger}+\right.$ H.c. $]$,

with $\omega_{\mathrm{c}}$ being the reservoir frequency, $\hat{a}_{r}$ being the annihilation operator of a photon, $\Omega_{0}$ being the amplitude of the drive, and $\omega_{\mathrm{d}}$ being the driving frequency. The local density-density coupling is given by Eq. (3) with $\hat{R}_{r}\left[\hat{R}_{r}^{\dagger}\right]$ replaced by $\hat{a}_{r}\left(\hat{a}_{r}^{\dagger}\right)$.

Assuming the reservoir to be in the steady state, one finds that the power spectrum of the noise is given by $[27,28]$

$$
S_{\mathrm{c}}(\omega)=\left|a_{0}\right|^{2} \frac{\kappa}{(\omega+\Delta)^{2}+\left(\frac{\kappa}{2}\right)^{2}},
$$

with $a_{0}=i \Omega_{0} /(i \Delta-\kappa / 2)$ being the classical amplitude of the field inside the cavity, $\kappa$ being the energy decay rate, and $\Delta=\omega_{\mathrm{d}}-\omega_{\mathrm{c}}$ is the detuning between the reservoir frequency and the drive.

As can be seen from Eq. (12), the sign of the detuning $\Delta$ determines whether $S_{\mathrm{c}}(\omega)$ peaks at either negative $(\Delta>0)$ or positive $(\Delta<0)$ frequency. Thus, substituting $S(\omega)$ by $S_{\mathrm{c}}(\omega)$ in Eq. (4) and choosing the detuning $\Delta$ to be negative while assuming $\omega_{\boldsymbol{k}}>\omega_{\boldsymbol{k}^{\prime}}$ leads to $\Gamma_{\boldsymbol{k} \rightarrow \boldsymbol{k}^{\prime}}>\Gamma_{\boldsymbol{k}^{\prime} \rightarrow \boldsymbol{k}}$ in Eq. (7). This corresponds to particles in high-energy modes being preferentially scattered into low-energy modes by having the reservoir absorb the excess of energy, leading to cooling [see Fig. 1(d)], which generalizes optomechanical cooling ideas $[27,28]$ to the many-body case. The reverse situation, with positive detuning $\Delta$, means the noise pumps energy incoherently into the array, scattering particles to higher energies. Another property of Eq. (4) that influences the dynamics described by Eq. (7) is the Bose enhancement factor; the rate at which a boson is scattered to a state with occupation $n$ is enhanced by a factor $n+1$.

Using the definition of the effective temperature, we find, in the limit where $\omega^{2} /\left[\Delta^{2}+(\kappa / 2)^{2}\right] \ll 1$,

$$
\beta_{\mathrm{eff}}(\omega)=\beta_{0}\left[1+\frac{\beta_{0}}{12 \Delta}\left(3+\beta_{0} \Delta\right) \omega^{2}\right]+\mathcal{O}\left(\omega^{4}\right),
$$

with the equilibrium temperature given by $\beta_{0}=-4 \Delta /\left[\Delta^{2}+\right.$ $\left.(\kappa / 2)^{2}\right]$.

\section{One-dimensional bosonic array}

For concreteness, we consider in the following a 1D array with

$$
\hat{H}_{\text {array }}=\omega_{0} \sum_{j=1}^{L} \hat{b}_{j}^{\dagger} \hat{b}_{j}+J \sum_{j=1}^{L-1}\left(\hat{b}_{j+1}^{\dagger} \hat{b}_{j}+\text { H.c. }\right)
$$
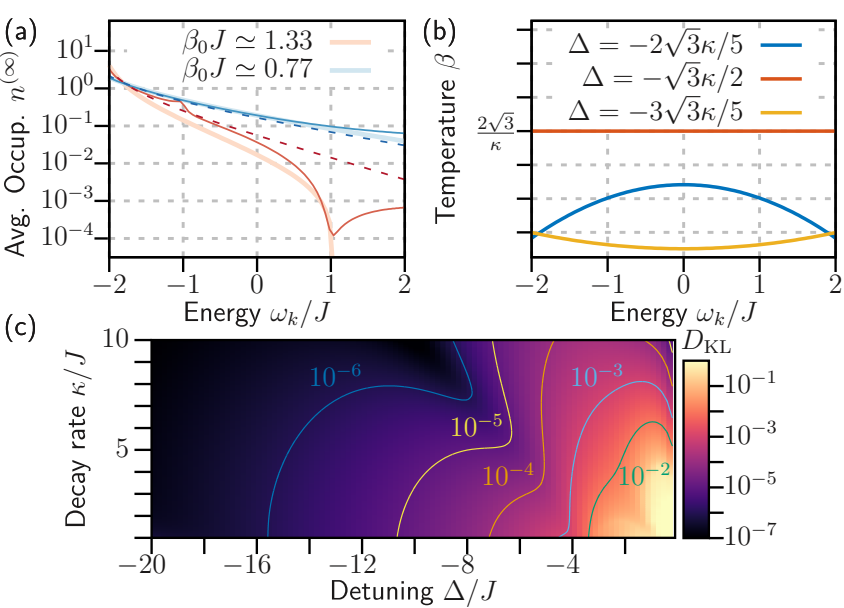

FIG. 2. Steady state of a 1D bosonic array with open-boundary conditions $\left(L=100, N=50, \chi=J / 1000\right.$, and $\Omega_{0}=J / 10$, unless specified otherwise). (a) Numerically observed steady-state distribution from Eq. (7) (solid thin lines), compared to perturbative solutions (solid thick lines) [see Eq. (15)], and Bose-Einstein statistics (dashed lines). (b) Effective energy-dependent inverse temperature $\beta\left(\omega_{k}\right)$ of the modified Bose-Einstein distribution. (c) KullbackLeibler divergence $D_{\mathrm{LK}}$ [see Eq. (17)] comparing the steady-state solution with the perturbative solution.

with $\omega_{0}$ being the frequency of the modes and $J$ being the hopping strength. The spectrum is given by $\omega_{k}=\omega_{0}+2 J \cos (k)$ and the density of states by $D(\omega)=1 /\left[2 \pi J \sqrt{1-(\omega / 2 J)^{2}}\right]$ for $|\omega| \leqslant 2 J$ and 0 otherwise.

In the long-time limit, the system approaches a steadystate distribution. In general, this does not correspond to any thermal equilibrium distribution, as it can be seen in our numerical results [see Fig. 2(a)] obtained on the basis of Eq. (7). However, we can characterize the final distribution using our perturbative treatment whenever $\beta_{0} J \ll 1$, since $\omega_{k} \propto J$. In particular, the latter condition is fulfilled whenever $J / \Delta \ll 1$ or $J / \kappa \ll 1$.

Substituting our series ansatz in Eq. (8) and looking for the steady state $\left[\dot{n}\left(\omega_{k}, t\right)=0\right]$, we find (see Appendix A)

$$
\begin{aligned}
n\left(\omega_{k}\right)= & n_{\mathrm{BE}}\left(\omega_{k}\right)\left\{1-\exp \left[\beta_{0}\left(\omega_{k}-\mu\right)\right] n_{\mathrm{BE}}\left(\omega_{k}\right)\right. \\
& \left.\times\left[\frac{\beta_{0}^{2}}{36 \Delta}\left(3+\beta_{0} \Delta\right)\left(\omega_{k}^{2}+18 J^{2}\right) \omega_{k}-\exp \left(-\beta_{0} \mu\right) c\right]\right\},
\end{aligned}
$$

with $c$ being a constant of integration. We can fix both $\mu$ and $c$ in Eq. (15) by enforcing particle number conservation, $\sum_{k} n\left(\omega_{k}\right)=N$; we first obtain $\mu$ by imposing normalization for $n_{\mathrm{BE}}$ and then extract $c$ from the same condition applied to the next order.

When we compare Eq. (15) to a generalized Bose-Einstein statistics with an energy-dependent inverse temperature [setting $\beta_{0} \rightarrow \beta\left(\omega_{k}\right)$ ], we conclude, to leading order,

$$
\beta\left(\omega_{k}\right)=\beta_{0}\left[1+\frac{\beta_{0}}{36 \Delta}\left(3+\beta_{0} \Delta\right)\left(\omega_{k}^{2}+18 J^{2}\right)\right] .
$$


The curvature of the effective temperature depends on the ratio between the detuning $\Delta$ and the decay rate $\kappa$ [see Fig. 2(b)]. Equation (16) in addition to reducing to $\beta_{0}$ for a small expansion parameter also reduces to $\beta_{0}$ at the special point $\Delta=-\sqrt{3} \kappa / 2$.

To assess the accuracy of our perturbative solution, we quantify its distance from the true steady-state solution of Eq. (15). We employ the Kullback-Leibler divergence [29] (relative entropy)

$$
D_{\mathrm{KL}}\left(p^{(\infty)} \| p\right)=\sum_{k} \frac{n^{(\infty)}\left(\omega_{k}\right)}{N} \log \left[\frac{n^{(\infty)}\left(\omega_{k}\right)}{n\left(\omega_{k}\right)}\right],
$$

which describes the amount of information lost when approximating one distribution by another. Here, we expressed our perturbative solution in terms of the normalized density $p\left(\omega_{k}\right)=n\left(\omega_{k}\right) / N$ [with $N$ being the total number of particles and $n\left(\omega_{k}\right)$ given by Eq. (15)] and compare it to the steady-state distribution $p^{(\infty)}\left(\omega_{k}\right)=n^{(\infty)}\left(\omega_{k}\right) / N$.

Figure 2(c) shows the Kullback-Leibler divergence as a function of detuning and decay rate. The numerical results were obtained by time evolving the kinetic equations until the system settles into the steady state.

We observe the perturbative solution in Eq. (15) approximating the nonequilibrium steady state well in a wide range of parameters. The approximation becomes better, as expected, in the limit $\beta_{0} J \ll 1$ [for $\beta_{0} J \rightarrow 0$, the effective temperature becomes energy-independent, and Eq. (15) reduces back to the Bose-Einstein distribution]. We stress that Eq. (15) is much closer to the real solution than the BoseEinstein distribution itself; indeed, in a large parameter regime we have $D_{\mathrm{KL}}\left(p^{(\infty)} \| p_{\mathrm{BE}}\right) / D_{\mathrm{KL}}\left(p^{(\infty)} \| p\right) \approx 10^{2}$ with $p_{\mathrm{BE}}=$ $n_{\mathrm{BE}} / N$ (see Appendix B).

We now discuss some distinct properties of the nonequilibrium distribution. In thermal equilibrium, detailed balance ensures that the Bose-Einstein distribution is independent of the density of states. This is no longer true here, out of equilibrium. As we show in Fig. 2(b), signatures of the density of states (with its characteristic divergence at the band edge in 1D) can be observed when the noise spectrum itself [see Eq. (4) with $S(\omega)=S_{\mathrm{c}}(\omega)$ ] is sufficiently narrow, i.e., for $\kappa / J \ll 1$. In that case, prominent features (almost nonanalytic) appear in the distribution at energies separated from the band edge by the detuning. These features are even more pronounced during the temporal dynamics (see below).

When deriving the kinetic equation [Eq. (4)], we pointed out the presence of the bosonic enhancement factors. The effects of such an enhancement can be observed when the particle density increases, particularly in the most strongly occupied regions of the band [see Fig. 3(a)]. For red detuning, we obtain many-particle ground-state cooling. To assess its efficiency, we plot in Fig. 3(b) the difference between the occupation of the single-particle ground state and the highest excited state in the band, $\delta n=n_{\text {high }}-n_{\mathrm{GS}}$. The bosonic enhancement serves to increase the efficiency of ground-state cooling.

In stark contrast to optomechanics, where the spectrum of the mechanical resonator is unbounded, we are dealing with a physical system that possesses a bound (many-particle) spectrum. This has an important consequence: Even for blue
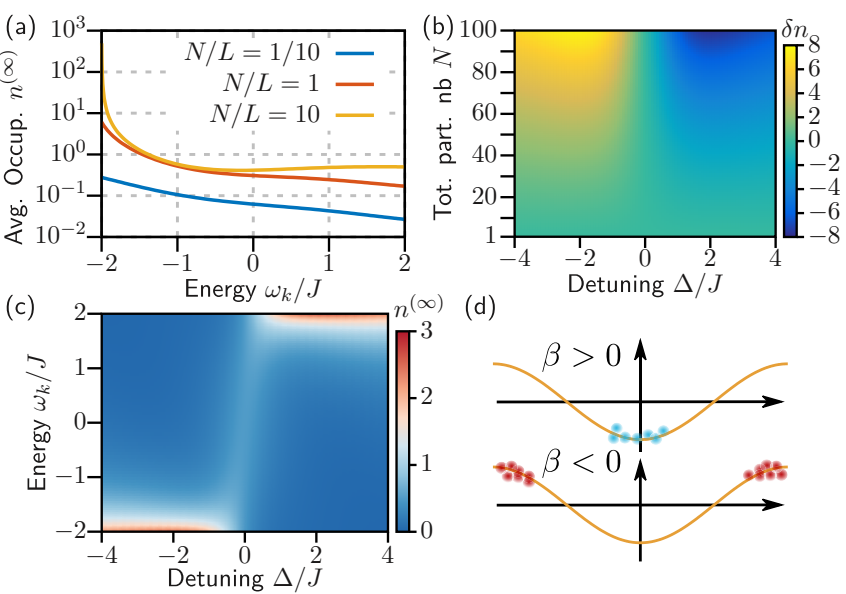

(d)

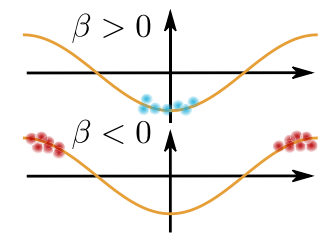

FIG. 3. Bosonic distribution in momentum space (long-time limit). (a) Impact of the Bose enhancement factor $(\Delta=-J, \kappa=3 J)$. As the particle density increases, the low-energy levels become more occupied during the cooling dynamics. (b) Efficiency of ground-state cooling. We plot the difference between the average occupation of the ground state and the highest excited state, $\delta n=n_{\mathrm{GS}}-n_{\text {high }}$ $(\kappa=3 J)$. (c) Transition toward negative temperature. For blue detuning $(\Delta>0)$, the system exhibits stable negative-temperatures steady states. (d) Schematic representation of states with positive and negative temperatures.

detuning $(\Delta>0)$, and without any recourse to nonlinear cavity dynamics, the system is still stable, as can be seen in Fig. 3(c). In this "heating" regime, particles accumulate at the upper band edge. Such states can be described by a negative temperature [see Fig. 3(d)]. Similar negative-temperature states were previously obtained using localized spin systems [30-32] and ultracold atoms trapped in optical lattices [33]. Creating stable negative states with optical lattices is challenging as it requires relatively complex state preparation. By contrast, here, one only needs to choose a positive detuning.

We noted earlier the existence of features in the steadystate distribution that are reminiscent of particle accumulation or depletion [see Fig. 2(b)]. As illustrated in Fig. 4, these features are substantially sharper and pronounced during the time evolution. To understand their origin, we consider the simplest possible situation: a uniform initial distribution. Such a uniform distribution in $k$ space can be realized, e.g., by incoherently loading the whole array with bosons at constant density (or quenching from a Mott insulator). At early times, the incipient deviations from the uniform distribution can be obtained perturbatively by considering Eq. (8) and substituting $n\left(\omega_{k}, t\right)$ by its initial uniform value $n_{0}$. Within this approximation, the integral in Eq. (8) is maximal (or minimal) when the divergence of the density of states aligns with the maximum of the spectrum $S(\omega)$ [or $S(-\omega)$ ]. Physically, this translates into having a large incoming rate of particles for states with $\omega_{k}=2 J+\Delta$ and a large outgoing rate for states with $\omega_{k}=-2 J-\Delta$. As a consequence, particles accumulate (deplete) at $\pm \Delta$ from the band edges [see Figs. 4(b) and 4(c)]. The accumulation of particles at $\omega_{k}=2 J+\Delta$ can subsequently lead to another accumulation of particles at $\omega_{k}=2 J+2 \Delta$ [see Fig. 4(b), $\Delta=-J / 2$ ]. As emphasized already above for the steady state, observing these extra peaks 

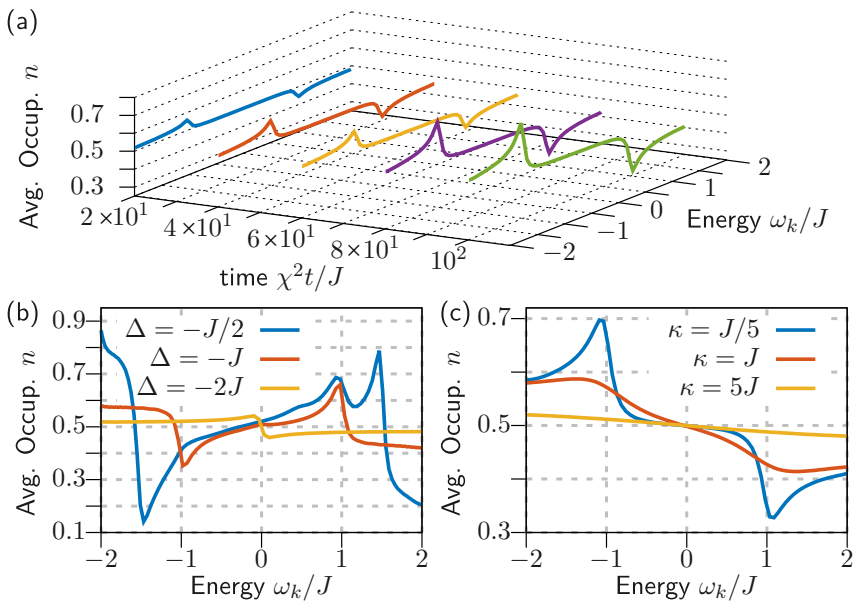

FIG. 4. Time evolution. (a) Snapshots of the distribution showing the emergence of accumulation and depletion of particles at $\pm \Delta$ off the band edges $(\Delta=-3 J, \kappa=J / 10)$. The steady-state distribution can be seen in Fig. 2(a). (b) Detuning dependence $\left(\chi^{2} t / J=10, \kappa=\right.$ $J / 10$ ). (c) Impact of the decay rate $\kappa$.

in the distribution is contingent on having a narrow cavity spectrum, with $\kappa \ll|\Delta|, J$ [see Fig. 4(c)].

In experimental implementations, two factors may come into play to distort the long-time dynamics from the idealized scenario. We mention them briefly, though a detailed study would be beyond the scope of the present work. First, there is the loss of array bosons at a constant rate, e.g., intrinsic cavity decay or mechanical dissipation. This will simply lead to an overall depletion of the total particle number $N(t) \sim e^{-\Gamma t}$; i.e., the steady state will slowly evolve, due to the modification of bosonic enhancement factors. Second, if there are interactions between the bosons, e.g., attractive interactions for a chain of transmon qubits instead of linear cavities, these will lead to additional scattering that is compatible with ground-state cooling but will drive the distribution closer to thermal.

\section{CONCLUSION}

We have developed a general framework to analyze both the short-time and long-time dynamics of a many-body system coupled to an engineered reservoir in a numberconserving way. In particular, we have established a perturbative technique to derive the nonequilibrium steady-state particle distribution. We have illustrated our theory by considering a $1 \mathrm{D}$ bosonic array with each site coupled to a driven cavity. However, our treatment is applicable to other nonequilibrium reservoirs coupled in a particle-conserving manner to arbitrary noninteracting many-body systems, i.e., in higher dimensions, for arbitrary lattices and band structures, and also for fermionic systems, with straightforward modifications.

\section{APPENDIX A: PERTURBATION THEORY}

In this appendix, we show in more detail how we obtained Eq. (15). As stated in Sec. III, the results were obtained by considering a 1D array with open-boundary conditions. In this case, the mode function is given by $\varphi_{\boldsymbol{r}}(\boldsymbol{k})=\varphi_{r}(k)=$ $\sqrt{2 /(L+1)} \sin (k r)$ with $r \in\{1, \ldots, L\}$ and $k=\pi j /(L+1)$ with $j \in\{1, \ldots, L\}$. With this mode function, we can evaluate the factor $\gamma_{k, k^{\prime}}=\gamma_{k, k^{\prime}}$ [see Eqs. (4) and (6)] using the result for a finite geometric series, and we find

$$
\gamma_{k, k^{\prime}}=\frac{1}{L+1}\left[1+\frac{1}{2} \delta_{k+k^{\prime}, \pi}\right],
$$

with $\delta_{m, n}$ being the Kronecker $\delta$ function.

To get the continuous model presented in the main text [see Eq. (8)], we approximate $\gamma_{k, k^{\prime}}$ by a constant factor, $\gamma_{k, k^{\prime}} \simeq$ $\gamma=1 /(L+1)$. This is possible because there is only one value of $k^{\prime}$ for a fixed $k$ such that $k+k^{\prime}=\pi$. Converting the sums in Eq. (7) of the main text to integrals, we find the equation for the steady state:

$$
\begin{aligned}
0= & \int_{-2 J}^{2 J} d \omega_{k^{\prime}} D\left(\omega_{k^{\prime}}\right)\left\{S(\omega) n\left(\omega_{k^{\prime}}\right)\left[n\left(\omega_{k}\right)+1\right]\right. \\
& \left.-S(-\omega) n\left(\omega_{k}\right)\left[n\left(\omega_{k^{\prime}}\right)+1\right]\right\} .
\end{aligned}
$$

To solve for $n\left(\omega_{k}\right)$, we start by expanding both $S(\omega)$ and $S(-\omega)$ in powers of $\beta_{0} \omega$. We have

$$
\begin{aligned}
S(\omega) & =\left|a_{0}\right|^{2} \frac{\kappa}{(\omega+\Delta)^{2}+\left(\frac{\kappa}{2}\right)^{2}} \\
& =\left|a_{0}\right|^{2} \frac{\kappa}{\Delta^{2}+\left(\frac{\kappa}{2}\right)^{2}} \frac{1}{1+\frac{4 \Delta}{\Delta^{2}+\left(\frac{\kappa}{2}\right)^{2}} \frac{\omega}{2}\left(1+\frac{\omega}{2 \Delta}\right)} \\
& =\left|a_{0}\right|^{2} \frac{\kappa}{\Delta^{2}+\left(\frac{\kappa}{2}\right)^{2}} \frac{1}{1-\beta_{0} \frac{\omega}{2}\left(1+\frac{\omega}{2 \Delta}\right)} \\
& =\left|a_{0}\right|^{2} \frac{\kappa}{\Delta^{2}+\left(\frac{\kappa}{2}\right)^{2}} \sum_{l=0}^{\infty}\left[\frac{\beta_{0}}{2 \omega}\left(1+\frac{\omega}{2 \Delta}\right)\right]^{l},
\end{aligned}
$$

and similarly we have

$$
S(-\omega)=\left|a_{0}\right|^{2} \frac{\kappa}{\Delta^{2}+\left(\frac{\kappa}{2}\right)^{2}} \sum_{l=0}^{\infty}\left[-\frac{\beta_{0}}{2 \omega}\left(1+\frac{\omega}{2 \Delta}\right)\right]^{l} .
$$

We also expand $n\left(\omega_{k^{\prime}}\right)=n\left(\omega_{k}+\omega\right)$ in a Taylor series around $\omega=0$, so we have

$$
n\left(\omega_{k}+\omega\right)=\sum_{l=0}^{\infty} \frac{n^{(l)}\left(\omega_{k}\right)}{l !} \omega^{l},
$$

where $n^{(l)}$ denotes the $l$ th derivative of $n\left(\omega_{j}\right)$.

By replacing Eqs. (A3), (A4), and (A5) into Eq. (A2), we can carry out the integral over the density of states. We look for solutions of the resulting equation in the form of a series expansion

$$
n\left(\omega_{k}\right)=\sum_{l=0}^{\infty} n_{l}\left(\omega_{k}\right),
$$

where we assume that $n_{l} \propto\left(\beta_{0} \omega_{k}\right)^{l}$.

By expanding Eqs. (A3), (A4), and (A5) to third order, i.e., up to $l=3$, we can find first-order differential equations for $n_{l}$ with $l \in\{0,1,2\}$ by grouping together terms proportional to $\left(\beta_{0} \omega_{k}\right)^{l}$. We note that we treat terms like $\left(\beta_{0} \omega_{k}\right) \omega_{k} / \Delta$ as being higher order; i.e., for this particular example, this term would be grouped together with terms scaling like $\left(\beta_{0} \omega_{k}\right)^{2}$. This is 

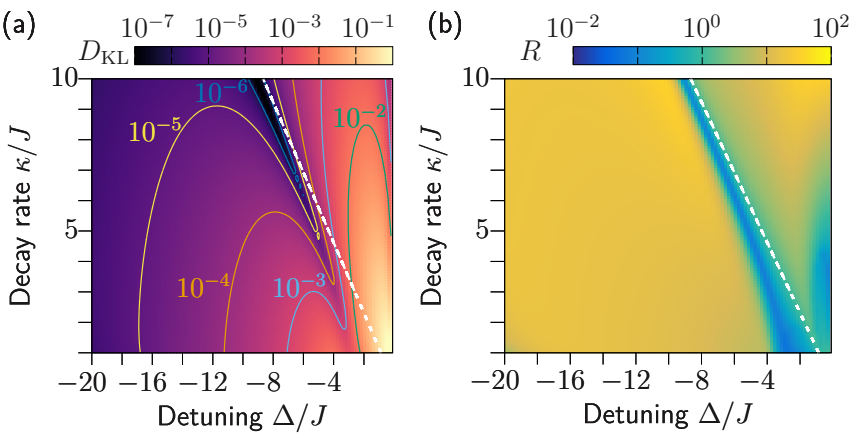

FIG. 5. (a) Kullback-Leibler divergence comparing the steadystate solution with the Bose-Einstein statistics given by Eq. (A8). (b) Ratio between $D_{\mathrm{KL}}\left(p^{(\infty)} \| p_{\mathrm{BE}}\right)$ and $D_{\mathrm{KL}}\left(p^{(\infty)} \| p\right)$. The white dashed line corresponds to $\Delta=-\sqrt{3} \kappa / 2$ where the temperature $\beta\left(\omega_{k}\right)$ is equal to $\beta_{0}$.

motivated by the result found for the effective temperature where $\beta_{2} / \beta_{0}=\beta_{0} /(4 \Delta)+\beta_{0}^{2} / 12$ (see main text).

Collecting terms proportional to $\beta_{0} \omega_{k}$, we find that $n_{0}\left(\omega_{k}\right)$ must obey the differential equation

$$
n_{0}^{\prime}\left(\omega_{k}\right)+\beta_{0} n_{0}\left(\omega_{k}\right)\left[n_{0}\left(\omega_{k}\right)+1\right]=0 .
$$

The solution of Eq. (A8) is the Bose-Einstein statistics with temperature $\beta_{0}$,

$$
n_{0}\left(\omega_{k}\right)=\frac{1}{\exp \left[\beta_{0}\left(\omega_{k}-\mu\right)\right]-1},
$$

and the constant of integration $\mu$ is the chemical potential, which is fixed by requiring that $\sum_{k} n_{0}\left(\omega_{k}\right)=N$.

Collecting terms proportional to $\left(\beta_{0} \omega_{k}\right)^{2}$, we find that $n_{1}\left(\omega_{k}\right)$ must obey the differential equation

$$
n_{1}^{\prime}\left(\omega_{k}\right)+\beta_{0} n_{1}\left(\omega_{k}\right)\left[n_{0}\left(\omega_{k}\right)+1\right]=0,
$$

whose solution is

$$
n_{1}\left(\omega_{k}\right)=c_{1} \frac{\exp \left[\beta_{0}\left(\omega_{k}-2 \mu\right)\right]}{\left\{\exp \left[\beta_{0}\left(\omega_{k}-\mu\right)\right]-1\right\}^{2}},
$$

with $c_{1}$ being a constant of integration. To determine the constant $c_{1}$, we require that $\sum_{k}\left[n_{0}\left(\omega_{k}\right)+n_{1}\left(\omega_{k}\right)\right]=N$. Note that we have previously fixed $\mu$ by requiring that $\sum_{k} n_{0}\left(\omega_{k}\right)=N$, which leads to $c_{1}=0$. We note that this procedure to find the constants of integration ensures that our solution always describes a distribution with $N$ particles at every order.

Finally, collecting terms proportional to $\left(\beta_{0} \omega_{k}\right)^{3}$, we find that $n_{2}\left(\omega_{k}\right)$ must obey the differential equation

$$
\begin{aligned}
& n_{2}^{\prime}\left(\omega_{k}\right)+\beta_{0} n_{2}\left(\omega_{k}\right)\left[2 n_{0}\left(\omega_{k}\right)+1\right]+\frac{\beta_{0}^{2}\left(3+\beta_{0} \Delta\right)\left(6 J^{2}+\omega_{k}^{2}\right)}{12 \Delta} \\
& \quad \times n_{0}\left(\omega_{k}\right)\left[n_{0}\left(\omega_{k}\right)+1\right]=0 .
\end{aligned}
$$

The solution of Eq. (A11) is

$$
\begin{aligned}
n_{2}\left(\omega_{k}\right)= & -\frac{\exp \left[\beta_{0}\left(\omega_{k}-\mu\right)\right]}{\left\{\exp \left[\beta_{0}\left(\omega_{k}-\mu\right)\right]-1\right\}^{2}} \\
& \times\left[\frac{\beta_{0}^{2}}{36 \Delta}\left(3+\beta_{0} \Delta\right)\left(18 J^{2}+\omega_{k}^{2}\right) \omega_{k}-\exp \left(\beta_{0} \mu\right) c_{2}\right],
\end{aligned}
$$

with $c_{2}$ being the constant of integration which is once more determined by the condition $\sum_{k}\left[n_{0}\left(\omega_{k}\right)+n_{2}\left(\omega_{k}\right)\right]=N$.

Combining Eqs. (A8), (A10), and (A12) together with the result $c_{1}=0$ leads to Eq. (15) of the main text. We note that the latter equation can predict negative occupation numbers, but this only occurs outside of the perturbative regime where the theory is not valid anymore.

\section{APPENDIX B: APPROXIMATING THE STEADY-STATE SOLUTION WITH THE BOSE-EINSTEIN STATISTICS}

In this appendix, we show that the third-order perturbative solution is a better approximation of the steady-state solution than the leading order Bose-Einstein statistics. In Fig. 5(a), we plot the Kullback-Leibler divergence between $p^{(\infty)}$ and $p_{\mathrm{BE}}$. We indicate by a white dashed line when $\beta\left(\omega_{k}\right)=\beta_{0}$ [see Eq. (16)], i.e., $\Delta=-\sqrt{3} \kappa / 2$. When this last condition is met, we have $n_{2}\left(\omega_{k}\right)=0$ if $c_{2}=0$.

In Fig. 5(b), we plot the ratio

$$
R=\frac{D_{\mathrm{KL}}\left(p^{(\infty)} \| p_{\mathrm{BE}}\right)}{D_{\mathrm{KL}}\left(p^{(\infty)} \| p\right)},
$$

which shows that the higher order perturbation approximates the steady-state solution more accurately than the leading order given by the Bose-Einstein statistics. We note, however, that in the close vicinity of $\Delta=-\sqrt{3} \kappa / 2$, the leading order is more suitable to approximate the steady state.
[1] J. F. Poyatos, J. I. Cirac, and P. Zoller, Quantum Reservoir Engineering with Laser Cooled Trapped Ions, Phys. Rev. Lett. 77, 4728 (1996).

[2] M. B. Plenio and S. F. Huelga, Entangled Light from White Noise, Phys. Rev. Lett. 88, 197901 (2002).

[3] E. Kapit, The upside of noise: Engineered dissipation as a resource in superconducting circuits, Quantum Sci. Tech. 2, 033002 (2017).

[4] H. Krauter, C. A. Muschik, K. Jensen, W. Wasilewski, J. M. Petersen, J. I. Cirac, and E. S. Polzik, Entanglement Generated by Dissipation and Steady State Entanglement of Two Macroscopic Objects, Phys. Rev. Lett. 107, 080503 (2011).
[5] K. W. Murch, U. Vool, D. Zhou, S. J. Weber, S. M. Girvin, and I. Siddiqi, Cavity-Assisted Quantum Bath Engineering, Phys. Rev. Lett. 109, 183602 (2012).

[6] S. Shankar, M. Hatridge, Z. Leghtas, K. M. Sliwa, A. Narla, U. Vool, S. M. Girvin, L. Frunzio, M. Mirrahimi, and M. H. Devoret, Autonomously stabilized entanglement between two superconducting quantum bits, Nature (London) 504, 419 (2013).

[7] Z. Leghtas, S. Touzard, I. M. Pop, A. Kou, B. Vlastakis, A. Petrenko, K. M. Sliwa, A. Narla, S. Shankar, M. J. Hatridge, M. Reagor, L. Frunzio, R. J. Schoelkopf, M. Mirrahimi, and M. H. Devoret, Confining the state of light to a quantum manifold by engineered two-photon loss, Science 347, 853 (2015). 
[8] J. T. Barreiro, M. Müller, P. Schindler, D. Nigg, T. Monz, M. Chwalla, M. Hennrich, C. F. Roos, P. Zoller, and R. Blatt, An open-system quantum simulator with trapped ions, Nature (London) 470, 486 (2011).

[9] Y. Lin, J. P. Gaebler, F. Reiter, T. R. Tan, R. Bowler, A. S. Sørensen, D. Leibfried, and D. J. Wineland, Dissipative production of a maximally entangled steady state of two quantum bits, Nature (London) 504, 415 (2013).

[10] D. Kienzler, H.-Y. Lo, B. Keitch, L. de Clercq, F. Leupold, F. Lindenfelser, M. Marinelli, V. Negnevitsky, and J. P. Home, Quantum harmonic oscillator state synthesis by reservoir engineering, Science 347, 53 (2015).

[11] E. E. Wollman, C. U. Lei, A. J. Weinstein, J. Suh, A. Kronwald, F. Marquardt, A. A. Clerk, and K. C. Schwab, Quantum squeezing of motion in a mechanical resonator, Science 349, 952 (2015).

[12] J.-M. Pirkkalainen, E. Damskägg, M. Brandt, F. Massel, and M. A. Sillanpää, Squeezing of Quantum Noise of Motion in a Micromechanical Resonator, Phys. Rev. Lett. 115, 243601 (2015).

[13] S. Diehl, A. Micheli, A. Kantian, B. Kraus, H. P. Büchler, and P. Zoller, Quantum states and phases in driven open quantum systems with cold atoms, Nat. Phys. 4, 878 (2008).

[14] F. Verstraete, M. M. Wolf, and J. Ignacio Cirac, Quantum computation and quantum-state engineering driven by dissipation, Nat. Phys. 5, 633 (2009).

[15] J. Cho, S. Bose, and M. S. Kim, Optical Pumping into ManyBody Entanglement, Phys. Rev. Lett. 106, 020504 (2011).

[16] F. Quijandría, D. Porras, J. J. García-Ripoll, and D. Zueco, Circuit QED Bright Source for Chiral Entangled Light Based on Dissipation, Phys. Rev. Lett. 111, 073602 (2013).

[17] E. Kapit, M. Hafezi, and S. H. Simon, Induced SelfStabilization in Fractional Quantum Hall States of Light, Phys. Rev. X 4, 031039 (2014).

[18] F. Reiter, D. Reeb, and A. S. Sørensen, Scalable Dissipative Preparation of Many-Body Entanglement, Phys. Rev. Lett. 117, 040501 (2016).

[19] S. Hacohen-Gourgy, V. V. Ramasesh, C. De Grandi, I. Siddiqi, and S. M. Girvin, Cooling and Autonomous Feedback in a Bose-Hubbard Chain with Attractive Interactions, Phys. Rev. Lett. 115, 240501 (2015).
[20] R. Ma, B. Saxberg, C. Owens, N. Leung, Y. Lu, J. Simon, and D. I. Schuster, A dissipatively stabilized Mott insulator of photons, Nature (London) 566, 51 (2019).

[21] J. Klaers, J. Schmitt, F. Vewinger, and M. Weitz, Bose-Einstein condensation of photons in an optical microcavity, Nature (London) 468, 545 (2010).

[22] J. Schmitt, T. Damm, D. Dung, F. Vewinger, J. Klaers, and M. Weitz, Observation of Grand-Canonical Number Statistics in a Photon Bose-Einstein Condensate, Phys. Rev. Lett. 112, 030401 (2014).

[23] M. Hafezi, P. Adhikari, and J. M. Taylor, Chemical potential for light by parametric coupling, Phys. Rev. B 92, 174305 (2015).

[24] M. Aspelmeyer, T. J. Kippenberg, and F. Marquardt, Cavity optomechanics, Rev. Mod. Phys. 86, 1391 (2014).

[25] J. C. Sankey, C. Yang, B. M. Zwickl, A. M. Jayich, and J. G. E. Harris, Strong and tunable nonlinear optomechanical coupling in a low-loss system, Nat. Phys. 6, 707 (2010).

[26] D. Jaksch, C. W. Gardiner, and P. Zoller, Quantum kinetic theory. II. Simulation of the quantum Boltzmann master equation, Phys. Rev. A 56, 575 (1997).

[27] A. A. Clerk, M. H. Devoret, S. M. Girvin, F. Marquardt, and R. J. Schoelkopf, Introduction to quantum noise, measurement, and amplification, Rev. Mod. Phys. 82, 1155 (2010).

[28] F. Marquardt, J. P. Chen, A. A. Clerk, and S. M. Girvin, Quantum Theory of Cavity-Assisted Sideband Cooling of Mechanical Motion, Phys. Rev. Lett. 99, 093902 (2007).

[29] S. Kullback and R. A. Leibler, On information and sufficiency, Ann. Math. Statist. 22, 79 (1951).

[30] E. M. Purcell and R. V. Pound, A nuclear spin system at negative temperature, Phys. Rev. 81, 279 (1951).

[31] A. S. Oja and O. V. Lounasmaa, Nuclear magnetic ordering in simple metals at positive and negative nanokelvin temperatures, Rev. Mod. Phys. 69, 1 (1997).

[32] P. Medley, D. M. Weld, H. Miyake, D. E. Pritchard, and W. Ketterle, Spin Gradient Demagnetization Cooling of Ultracold Atoms, Phys. Rev. Lett. 106, 195301 (2011).

[33] S. Braun, J. P. Ronzheimer, M. Schreiber, S. S. Hodgman, T. Rom, I. Bloch, and U. Schneider, Negative absolute temperature for motional degrees of freedom, Science 339, 52 (2013). 